\title{
Research training in urology
}

\author{
Paul H Lange
}

In considering the topic of research training in urology, one must address two major questions. In today's world, should surgeons still aspire to life long careers in research? If so, how can this be done?

Formerly it was axiomatic that surgeons could succeed at scientific inquiry. Their contributions often changed both medicine and basic biology. In many cases these advances were made first in the laboratory and then in the operating room by action-orientated personalities trying to save a life. Indeed, nine surgeons have received Nobel prizes, including two urologists, one of whom was Charles Huggins for his work in prostate cancer. Prostate cancer is one of many areas of genitourinary medicine in which most of the major advances have been made by urologists, their associates, or both.

Today the demands of a career in medical research are greater, especially for the aspiring surgeon/scientist. A working knowledge of molecular biology is desirable, but requires many preparatory years, often in research environments alien to clinicians. Some have suggested that modern surgeon/scientists focus exclusively on careers as clinical trialists or experts in population science. I disagree; for optimal translational progress in medicine it is essential that some surgeon/scientists have 'wet lab' experience. Other barriers more unique to the surgeon than the medicine physician include the length and rigor of residency and fellowship training, and the fact that maintenance of surgical skills requires more time.

Both physicians and surgeons must participate in medical inquiry, for only they can truly bring the wonderful opportunities of modern biology to the bedside. Surgeons in particular have a unique insight into the patient, the affected organ, and the disease. They also often exhibit more of a 'can do' approach. Medical history is replete with examples where that insight and attitude made all the difference.

I believe there are four phases to the development of urological surgeon/scientists.
Both

physicians and surgeons must participate in medical inquiry, for only they can truly bring the wonderful opportunities of modern biology to the bedside.

\section{PH Lange is}

Chairman of the

Department of Urology at the

University of

Washington,

Seattle.

\section{Competing interests}

The author declared he has no competing interests.

www.nature.com/clinicalpractice doi:10.1038/ncpuro0149
The 'excitement phase', which lasts around 1 year, is when an individual either does or does not get excited about research. This should occur during or before residency, or in early fellowship training. It is desirable for all urology residents have this exposure so as to optimize the surgeon/scientist pool and to improve the practicing urologist, although I am not sure this is currently feasible for many training programs.

The 'consolidation phase', lasting 2 years or occurring in the second consecutive year after the year of the excitement phase, is when the motivated individual accumulates the kudos and data to begin writing grants.

The 'establishment phase' lasts 5 years or more. Here research activity consumes initially up to $80 \%$, and never less than $50 \%$, of the time. This is the 'make or break' period where a person joins the academic staff, gains funding for a salary and research support, obtains independent research space, and makes significant contributions to the field. During this time a critical task for surgeon/ scientists is to establish a solid association with a research partner, such as an individual with a PhD, or a laboratory, who assumes more responsibility for the day-to-day operations of research as the surgeon/scientist's clinical activity grows.

The final phase is known as the 'transfer phase'. This is when the established investigator and/or laboratory serves as a training ground for, and gives inspiration to, other aspiring surgeon scientists.

In order to effectuate these developmental phases, training curriculums will have to change. Subspecialization and its requisite fellowship training will need more embellishment and legitimacy. Funding for, and promotion of, the aspiring urological surgeon/scientist, especially during the establishment phase, will need more attention. I have been encouraged that leaders in urology and the greater research community are recognizing these challenges and seeking ways to solve them. At stake is not only the survival of urology as a vibrant specialty, but more importantly the optimal welfare of the patient with urologic disease. 